\title{
ARTICLE
}

\section{Irisin exerts a therapeutic effect against myocardial infarction via promoting angiogenesis}

\author{
Qiao Liao ${ }^{1,2,3}$, Shuang $\mathrm{Qu}^{1,2,3}$, Lu-xun Tang ${ }^{1,2,3}$, Liang-peng $\mathrm{Li}^{1,2,3}$, Duo-fen $\mathrm{He}^{1,2,3}$, Chun-yu Zeng ${ }^{1,2,3}$ and Wei Eric Wang ${ }^{1,2,3}$
}

Irisin, a myokine, is cleaved from the extracellular portion of fibronectin domain-containing 5 protein in skeletal muscle and myocardium and secreted into circulation as a hormone during exercise. Irisin has been found to exert protective effects against lung and heart injuries. However, whether irisin influences myocardial infarction (MI) remains unclear. In this study we investigated the therapeutic effects of irisin in an acute MI model and its underlying mechanisms. Adult C57BL/6 mice were subjected to ligation of the left anterior descending coronary artery and treated with irisin for 2 weeks after Ml. Cardiac function was assessed using echocardiography. We found that irisin administration significantly alleviated Ml-induced cardiac dysfunction and ventricular dilation at 4 weeks post-MI. Irisin significantly reduced infarct size and fibrosis in post-MI hearts. Irisin administration significantly increased angiogenesis in the infarct border zone and decreased cardiomyocyte apoptosis, but did not influence cardiomyocyte proliferation. In human umbilical vein endothelial cells (HUVEC), irisin significantly increased the phosphorylation of ERK, and promoted the migration of HUVEC detected in wound-healing and transwell chamber migration assay. The effects of irisin were blocked by the ERK inhibitor U0126. In conclusion, irisin improves cardiac function and reduces infarct size in post-MI mouse heart. The therapeutic effect is associated with its pro-angiogenic function through activating ERK signaling pathway.

Keywords: irisin; myocardial infarction; angiogenesis; ERK signal pathway; U0126

Acta Pharmacologica Sinica (2019) 40:1314-1321; https://doi.org/10.1038/s41401-019-0230-z

\section{INTRODUCTION}

Acute myocardial infarction (MI) occurs as a result of insufficient myocardial perfusion, leading to a massive, irreversible loss of myocardial cells, which is a major threat to human health worldwide [1]. Despite optimal treatments, including medication, percutaneous coronary intervention and coronary artery bypass grafting, MI develops to pathological cardiac remodeling and heart failure. To combat this profile of progressive cardiac deterioration after $\mathrm{Ml}$, new approaches have emerged with emphasis on ameliorating fibrotic remodeling, increasing angiogenesis and preserving or replacing cardiomyocytes [2].

Exercise training confers sustainable protection against ischemia-reperfusion injury in animal models and has been associated with improved survival following a heart attack in humans [3]. Several myokines are produced by skeletal muscle, and changes in myokine levels following Ml were investigated. Irisin, which is secreted by skeletal muscle and myocardium into circulation during exercise, is proteolytically cleaved and secreted as the hormone peptide from fibronectin domain-containing 5 (FNDC5) [4]. A negative correlation between serum irisin and the severity of the MI was observed in exercise-trained rats [5]. However, the protective mechanisms are not known.

Irisin appears to be a multifunctional peptide involved in cardiovascular disease $[6,7]$. Irisin could protect against pressure overload-induced cardiac hypertrophy by inducing protective autophagy [6]. In addition, irisin protects cardiomyocytes against hypoxia and reoxygenation injury and the heart against ischemiareperfusion (I/R) injury [7]. Irisin generated a protective effect against myocardial $\mathrm{I} / \mathrm{R}$ injury by suppressing the opening of the mitochondrial permeability transition pore [8] through a SOD2dependent mitochondria mechanism [9]. The protective effects of irisin have also been found in endothelial cells. Irisin significantly alleviates endothelial dysfunction and decreases endothelial apoptosis in diabetic mice by reducing oxidative stress $[10,11]$. We previously reported that limb remote ischemic preconditioning (RIPC) releases irisin to protect against I/R injury to the lung and that irisin facilitates RIPC-mediated lung protection [12]. RIPC is also an effective means of protection against $\mathrm{Ml}$ to hearts. However, the therapeutic effect of irisin on MI remains unclear. The present study therefore tested the hypothesis that administration of recombinant irisin protects heart from $\mathrm{Ml}$ injury and ameliorates cardiac remodeling after MI.

\section{MATERIALS AND METHODS}

Mouse model of myocardial infarction

Adult C57/BL6 male mice (SPF Biotechnology, Beijing, China) were subjected to $\mathrm{Ml}$ by ligation of the left anterior descending (LAD) coronary artery, as previously described [13]. In brief, 8-week-old mice were anaesthetized with $5 \%$ isoflurane in an airtight

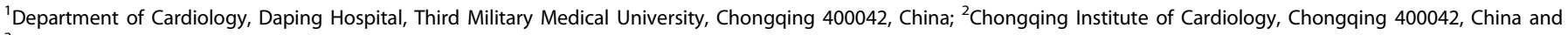
${ }^{3}$ Chongqing Cardiovascular Clinical Research Center, Chongqing 400042, China

Correspondence: Wei Eric Wang (weiericwang@163.com) or Chun-yu Zeng (chunyuzeng01@163.com)

These authors contributed equally: Qiao Liao, Shuang Qu.
}

Received: 28 December 2018 Accepted: 14 March 2019

Published online: 6 May 2019 
chamber and placed in a supine position on a heating pad $\left(37^{\circ} \mathrm{C}\right)$. Following tracheal intubation, mice were artificially ventilated using a volume-controlled ventilator with $2.4 \%$ isoflurane. Following skin incision, lateral thoracotomy at the third intercostal space was performed by blunt dissection of the intercostal muscles. The LAD artery was ligated using a 7-0 prolene suture. Subsequently, thoracic wall incisions were sutured with 6.0 non-absorbable silk sutures, and the wound was closed using Vetbond ( $3 M, M N)$ skin adhesive. Mice were then warmed for several minutes until recovery. After Ml surgery, irisin $(5 \mu \mathrm{g} / \mathrm{g}$ every day, Cat. No. 067-17, Phoenix Pharmaceuticals, CA) [14] and U0126 (3 $\mu \mathrm{g} / \mathrm{g}$ every day, Cat. No. HY-12031, MedChemExpress, NJ) [15] were injected intraperitoneally per day for 2 weeks. This study was approved by the Research Council and Animal Care and Use Committee of Daping Hospital, Third Military Medical University. All experiments conformed to the guidelines of the American Association for the Accreditation of Laboratory Animal Care.

\section{Echocardiography}

Cardiac function was evaluated by transthoracic echocardiography (GE vivid 9 dimension, General Electric Company, Fairfield, CT) following the methods we described previously [16]. Briefly, hearts were viewed in the short-axis with M-mode by averaging results from three consecutive heartbeats. Diastolic left ventricle internal diameter (LVIDd) and systolic left ventricle internal diameter (LVIDs) were measured to determine structural changes in cardiac morphology. Fractional shortening (\%FS) was calculated as (LVIDd - LVIDs)/LVIDd $\times 100$. Left ventricle ejection fraction (\%EF) was automatically calculated according to the Teicholz formula. All echocardiography measurements were performed in a blind manner.

Measurement of myocardial infarct size

The infarct size after injury for 4 weeks was defined by phosphate-buffered $1 \%$ 2,3,5-triphenyltetrazolium chloride (TTC) staining as previously described [17]. In brief, mice were anesthetized and transcardially perfused of PBS followed by $10 \mathrm{~mL}$ of $1 \%$ TTC (Cat. No. 362883, Sigma, St. Louis, MO) for $10 \mathrm{~min}$. After transcardial TTC perfusion, the heart was cut into five 1-mm thick transverse slices from the apex to the base parallel to the atrioventricular groove. Then, the hearts of mice were placed into 4\% paraformaldehyde (PFA, Cat. No. E672002, Sangon Biotech, Shanghai, China). The infarct size was delineated and calculated using ImageJ software.

\section{Histology}

Mouse heart tissues were harvested and fixed in 4\% PFA/PBS solution overnight at room temperature and then processed for paraffin sections. For analysis of cardiac regeneration and fibrotic area following myocardial infarction procedure or drug injection, paraffin sections were cut through the entire ventricle from apex to base into serial sections at $0.5-\mathrm{mm}$ intervals. After the dewaxing procedure, Masson's trichrome staining (Cat. No. G1345, Solarbio, Beijing, China) was performed according to standard procedures. Scar size was calculated relative to left ventricle size by ImageJ software.

\section{Apoptosis detection}

To detect the effect of irisin on cardiomyocyte apoptosis, the terminal deoxynucleotidyl transferase mediated dUTP nick-end labeling (TUNEL) assay was performed at 2 days post-MI as we previously described [17]. In brief, after the dewaxing procedure, the sections were stained with anti-tropomyosin antibody (1:100, Cat. No. T9283, Sigma), anti-cardiac troponin T antibody (1:100, Cat. No. MA5-12960, Invitrogen, Thermo Fisher Scientific, MA) and 4',6-diamidino-2-phenylindole dihydrochloride (DAPI, Cat. No. C0065, Solarbio). TUNEL assay was performed using the In Situ Cell Death Detection Kit (Cat. No. 11684817910, Roche Applied Bio
Sciences, Switzerland) according to the manufacturer's protocols. The slices were observed with a fluorescence microscope (Nikon, Tokyo, Japan). The percentage of apoptotic nuclei per section was calculated by counting the number of TUNEL-staining cardiomyocyte nuclei divided by the total number of DAPI-positive nuclei. We also calculated the percentage of apoptosis in noncardiomyocytes per section. The numbers of apoptotic nuclei were counted in ten non-adjacent images of peri-infarction areas, and a total of five sections per animal were analyzed.

\section{Immunofluorescence analysis}

Formalin-fixed hearts were processed, embedded in paraffin, and cut into $5-\mathrm{mm}$ thick sections. The sections were blocked with $5 \%$ bovine serum albumin (BSA, Sangon Biotech) in PBS containing $0.1 \%$ Triton $\mathrm{X}-100$ for $1 \mathrm{~h}$ at room temperature. The sections were incubated with primary antibodies overnight at $4{ }^{\circ} \mathrm{C}$, subsequently washed thrice with PBS and incubated with corresponding secondary antibodies conjugated to Alexa Fluor 488 or 555 (Invitrogen, Cat. No. A-11001, A-21422, A-21428, Thermo Fisher Scientific, MA) for $2 \mathrm{~h}$ at $37^{\circ} \mathrm{C}$. The slides were mounted in antifade mounting medium. The following primary antibodies were used: anti-Ki67 antibody (1:100, Cat. No. D3B5, Cell Signaling Technology, MA), anti-cardiac troponin T antibody (cTnT, 1:100, Cat. No. MA5-12960, Invitrogen), anti-a-smooth muscle actin antibody (anti-a-SMA, Cat. No. ab125884, 1:100, Abcam, Cambridge, UK) and anti-CD31 antibody (1:100, Cat. No. ab28364, Abcam). DAPI was used for nuclear staining. Angiogenesis was detected by calculating the capillary density per field in the infarct border zone, as we previously reported [18]. For the quantification of the number of $\mathrm{Ki}^{+} 7^{+}$cardiomyocytes, the results acquired from sections of the heart harvested from each animal with at least 5 different fields and positions for each section were averaged. In all cell-counting experiments, fields of view were randomized to reduce counting bias.

Capillary-like tube formation assay

Human umbilical vein endothelial cells (HUVECs) were purchased from American Type Culture Collection. Cells were seeded at a density of $5 \times 10^{3} / \mathrm{cm}^{2}$ flask DMEM (Gibco, Cat.No.10569044, Thermo Fisher Scientific) supplemented with $10 \%$ fetal bovine serum (FBS, Cat. No.10099-141, Gibco) and 1\% penicillin/ streptomycin (Cat. No. P1400, Solarbio). Cells were maintained at $37^{\circ} \mathrm{C}$ in $5 \% \mathrm{CO}_{2}$, and the media were refreshed every third day. Cells were starved in DMEM containing $1 \%$ FBS for $24 \mathrm{~h}$ before irisin treatment $(200 \mathrm{ng} / \mathrm{mL})$. Pictures of capillary-like tube formation were obtained with a computer-assisted microscope. HUVEC tube formation assays are used as a model for studying endothelial tube formation in vitro [19].

\section{Cell migration assay}

A scratch wound migration assay was performed as previously described [19]. In brief, HUVECs were seeded in a 6-well plate in normal growth media for $48 \mathrm{~h}$. Media were then changed to DMEM containing $1 \%$ FBS for $24 \mathrm{~h}$. For each well, two parallel scratches were made within the cell layer using a sterile $100-\mu \mathrm{L}$ pipette tip, and then HUVECs were treated with irisin. The scratch was visualized using a Nikon inverted microscope(Tokyo, Japan). To calculate cell migration, HUVECs that migrated into the wounded area from the border were photographed and measured in each image to yield an average value for each condition compared with the time zero point.

Cell migration was also analyzed using a transwell migration assay [19]. In brief, cells were plated into the top well of transwell migration chamber (Merck, Cat. No. MMA205, Darmstadt, Germany). After 24-h treatment with irisin, the nonmigrating cells were removed with a cotton swab, and the migrating cells on the underside of membrane were stained with crystal violet. 
a1

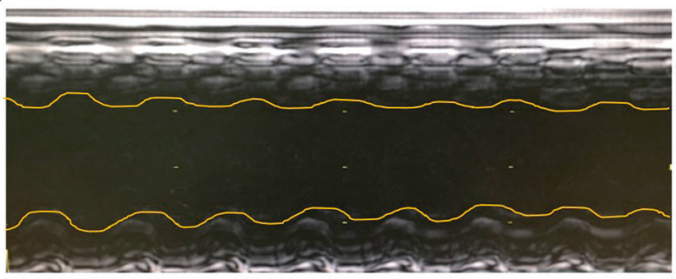

MI

a2

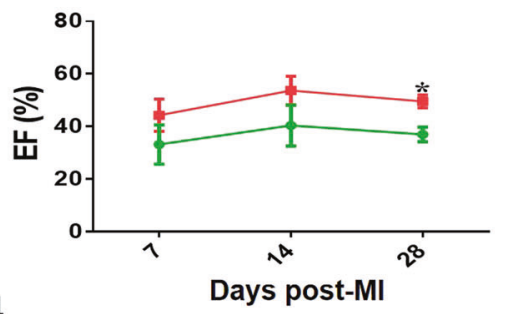

a4

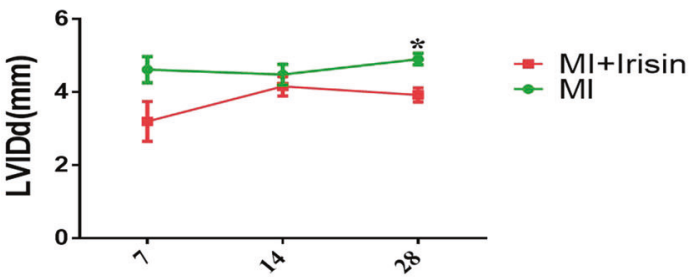

Days post-MI

b1
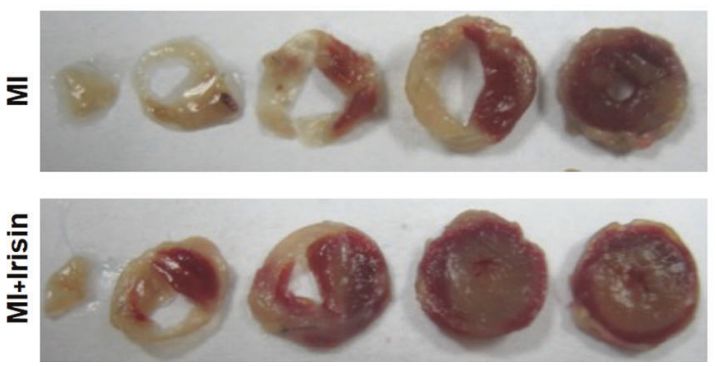

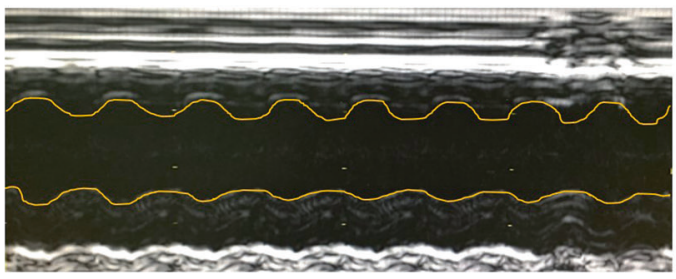

Ml+Irisin a3

a5
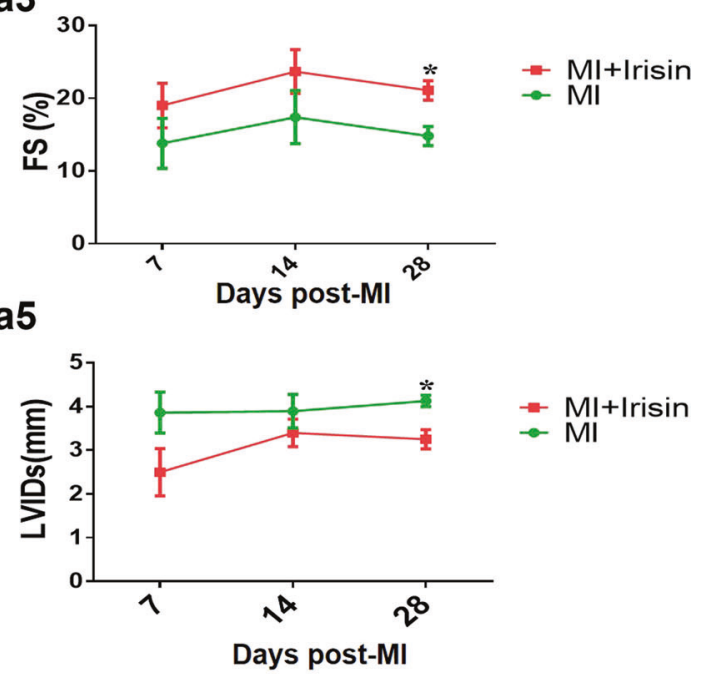

b2

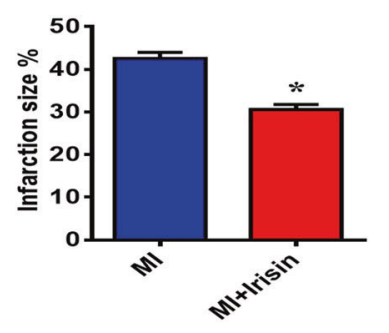

Fig. 1 Irisin improved cardiac function and reduced infarct size in post-MI mice. a1 Representative images of M-mode echocardiographic images at 28 days post-MI. a2-5 The left ventricle ejection fraction (EF), fractional shortening (FS), diastolic left ventricle internal diameter (LVIDd) and systolic left ventricle internal diameter (LVIDs) after MI at 7, 14, and 28 days post-MI. b Representative images and quantification of infarct size analyzed by TTC staining. $n=12,{ }^{*} P<0.05$ vs. control

Western blot analysis

Protein was extracted from HUVECs after treatment with irisin $(200 \mathrm{ng} / \mathrm{mL})$, U0126 $\left(10^{-5} \mathrm{~mol} / \mathrm{L}\right)$, irisin + U0126, or PBS. Protein was extracted from mouse heart post-MI after treatment with irisin, U0126, irisin + U0126 or PBS. Western blotting was performed as previously reported [20]. Proteins from heart tissue lysates containing $50 \mu \mathrm{g}$ of protein were separated by SDS-PAGE and electrophoretically transferred onto NC membranes (Bio-Rad, CA). After treatment with blocking buffer, the blots were probed with the anti-phospho ERK antibody (1:1000, Cat. No. 4370 S, Cell Signaling Technology), anti-ERK antibody (1:1000, Cat. No. $4395 \mathrm{~S}$, Cell Signaling Technology) and $\beta$-actin antibody (1:600, Santa Cruz Biotechnology, CA) at $4^{\circ} \mathrm{C}$ overnight. Membranes were washed in TBST and incubated with the appropriate secondary antibodies (Li-Cor, IRDye 800CW, 1:10 000) for $2 \mathrm{~h}$ at room temperature. Membranes were washed and visualized with an Odyssey Imaging System.
Statistical analysis

Data are expressed as the means \pm SEM. All data collected and analyzed were assumed to be distributed normally. Comparison within groups was made by repeated measures ANOVA (or paired $t$ test when only 2 groups were compared), and comparison among groups (or $t$ test when only 2 groups were compared) was made by factorial ANOVA using the Holm-Sidak test. A value of $P<0.05$ was considered statistically significant.

\section{RESULTS}

Irisin improved cardiac function and reduced infarct size in postMI hearts

To determine if irisin regulates cardiac remodeling postischemia injury, eight-week-old C57/BL6 mice were subjected to MI by ligation of left anterior descending (LAD) coronary artery. After MI injury, mice were injected with irisin [14] or PBS 
a1
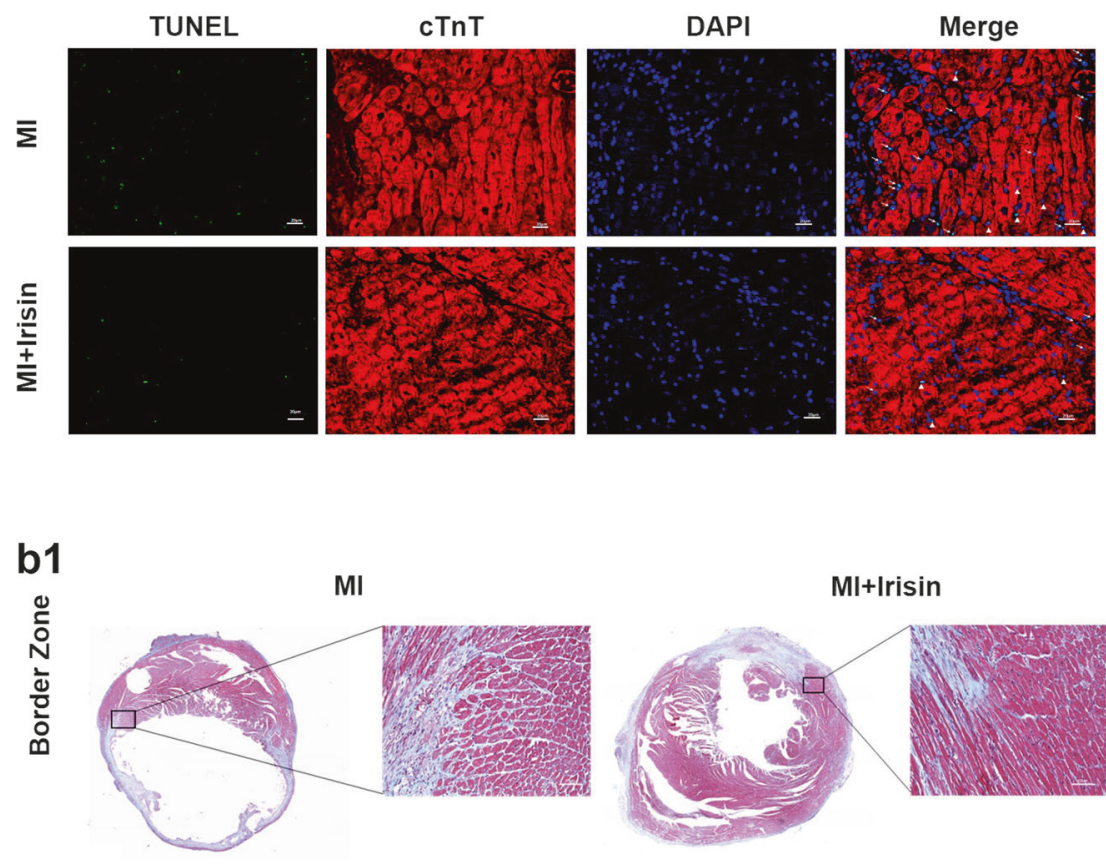

a2

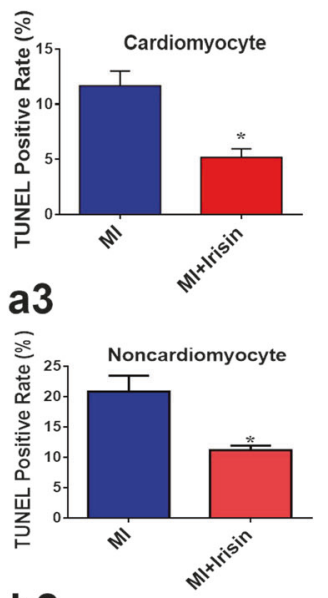

b2
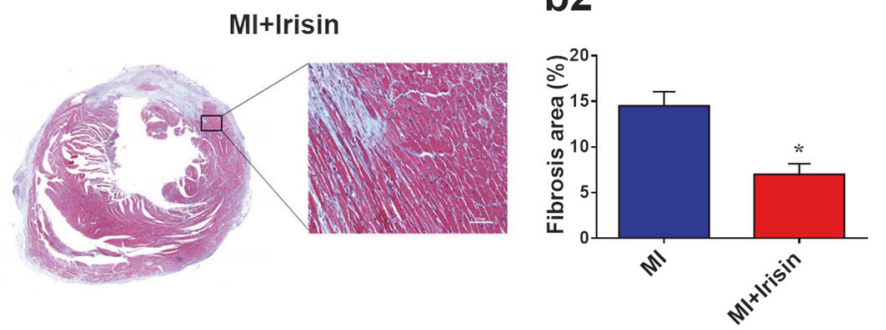

c2
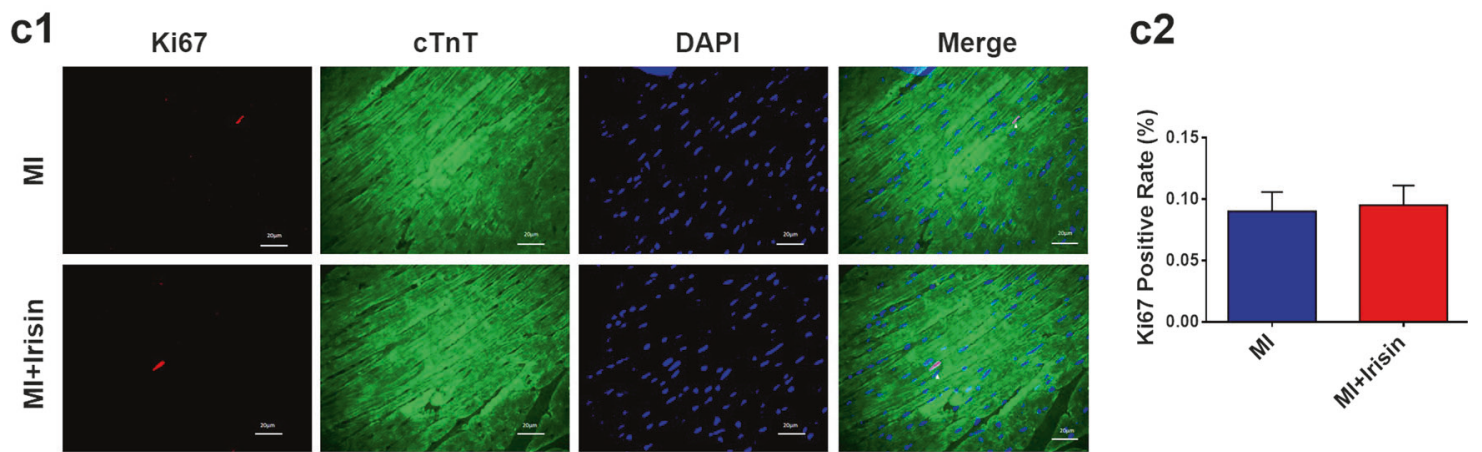

Fig. 2 Irisin decreased cardiomyocyte apoptosis and fibrosis in post-MI hearts. a Representative images and quantification of apoptosis indicator TUNEL-positive cardiomyocytes and noncardiomyocyte in heart tissue at 2 days post-MI. Cardiomyocytes were indicated with cTnT staining, and nuclei were stained with DAPI, scale bar $=20 \mu \mathrm{m}$. A arrow indicates noncardiomyocyte; a triangle indicates cardiomyocyte. b Representative images and quantification of fibrosis of infarct border zone at 4 weeks post-Ml detected with Masson trichrome staining. Scale bar $=50 \mu \mathrm{m}$. c Representative images and quantification of cardiomyocyte proliferation at 7 days post-Ml, indicated by Ki67 immunostaining. $n=6$. scale bar $=20 \mu \mathrm{m}$. ${ }^{*} P<0.05$ vs. control

intraperitoneally per day for 2 weeks. Cardiac function was evaluated by echocardiographic analysis at 7 days, 14 days and 28 days post-MI. The results showed that at day 7 and day 14, irisin treatment moderately increased EF and FS and moderately decreased LVIDd and LVIDs, but there were no significant differences (Fig. 1a). However, at day 28 post-MI, irisin mice had significantly improved LVFS and LVEF compared with control mice (Fig. 1a). Meanwhile, irisin-treated mice had significantly smaller LV chamber size than control mice, as indicated by the significant reduction in LVIDd and LVIDs at day 28 post-MI (Fig. 1a). Meanwhile, TTC staining revealed that irisin significantly reduced infarct size compared with the control at day 28 post-MI (Fig. 1b). These data indicated that irisin could improve cardiac function, limit ventricular dilation and reduce infarct size in post-MI hearts.
Irisin decreased cardiomyocyte apoptosis and fibrosis in post-MI hearts

Irisin has been previously reported to protect cells from apoptosis [21] and promote cell proliferation [22, 23]. We next detected fibrosis and cardiomyocyte proliferation in hearts. Cell apoptosis was detected at 2 days post-MI with TUNEL staining. Cardiomyocytes were indicated by CTnT staining. The data showed that irisin treatment significantly reduced TUNEL-positive cells in both cardiomyocytes and noncardiomyocytes. It suggested that irisin preserved myocardium at least partially through repressing cardiomyocyte apoptosis (Fig. 2a).

Masson's trichrome staining was performed to determine fibrosis at day 28 post-Ml. The result showed that irisin significantly decreased fibrosis in the peri-infarct border zone compared with the control (Fig. 2b). Cardiomyocyte proliferation 

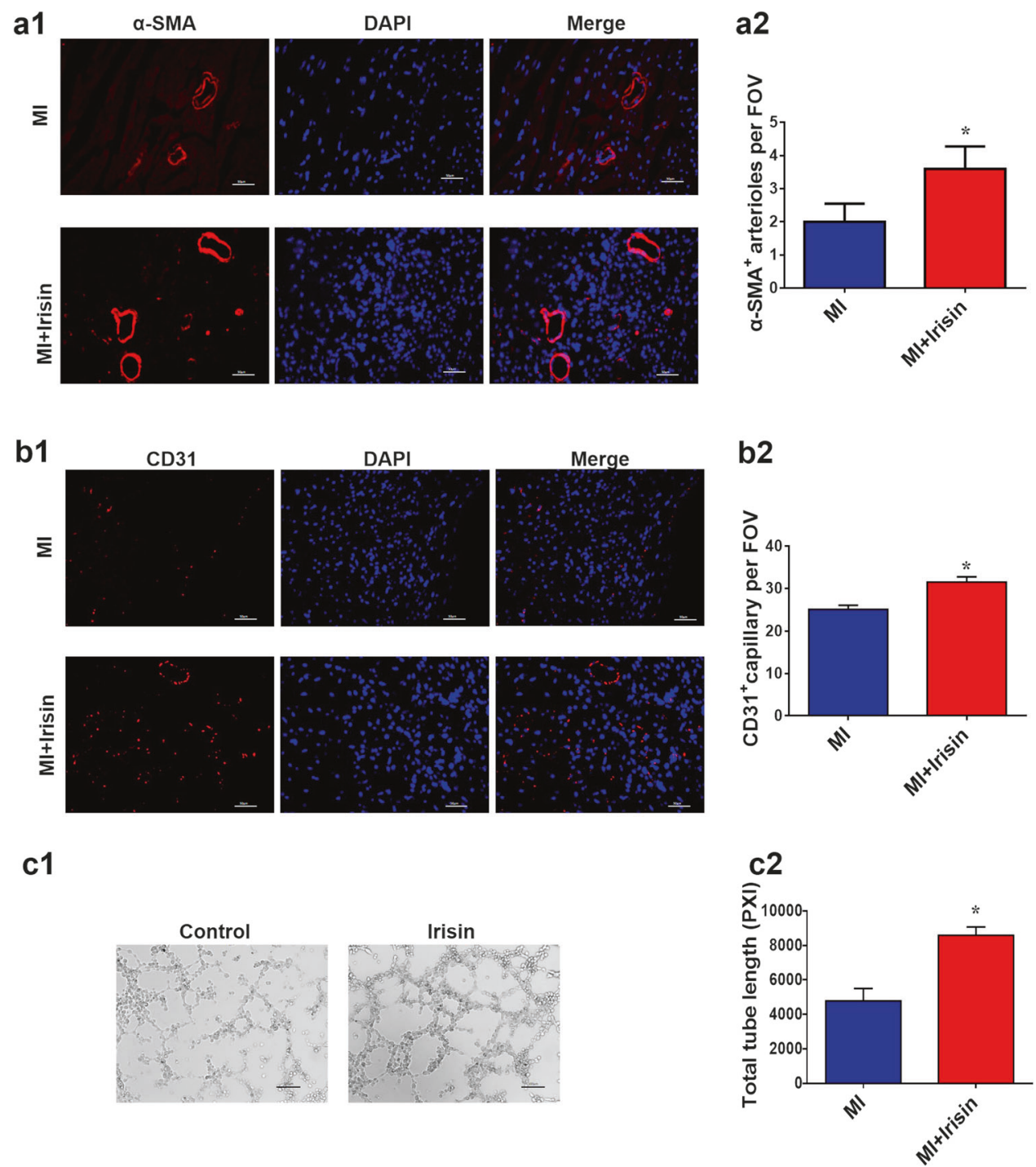

Fig. 3 Irisin promoted angiogenesis in post-MI hearts. a Representative angiogenesis images and quantification of $\alpha$-SMA ${ }^{+}$arterioles per FOV (field of view) ratio in the infarct border zone with immunostaining of the smooth muscle cells, $n=6$, scale bar $=50 \mu \mathrm{m}$. $\mathbf{b}$ Representative angiogenesis images and quantification of $\mathrm{CD} 31^{+}$capillary per FOV ratio in the infarct border zone with immunostaining of endothelial marker CD31 $n=6$, scale bar $=50 \mu \mathrm{m},{ }^{*} P<0.05$ vs. control. c Representative images of endothelial cell tube formation assay with HUVECs in vitro. $n=3$, scale bar $=100 \mu \mathrm{m},{ }^{*} P<0.05$ vs. control

was detected by immunofluorescent staining of the cell cycle marker Ki67. The results showed that the rate of $\mathrm{Ki}^{+} 7^{+}$ cardiomyocytes was comparable between the irisin- and PBStreated groups (Fig. 2c), indicating that irisin did not influence cardiomyocyte proliferation in post-MI heart.

Irisin promoted angiogenesis in post-MI hearts

Previous studies have suggested that irisin induces angiogenesis in HUVECs in vitro [24] and in zebrafish embryos in vivo [25]. Insufficient post-Ml angiogenesis has been identified as a nonnegligible event that precipitates heart failure progression. On the other hand, boosted angiogenesis ameliorated cardiac remodeling post-MI [26]. Therefore, we next detected angiogenesis and arteriogenesis in hearts with CD31 and a-SMA staining analysis, which are widely used markers for angiogenesis quantification. Heart tissues at 14 days post-MI were immunostained, and the results showed that CD31 and a-SMA expression in the border zone were increased in irisin-treated hearts (Fig. 3a, b). In in vitro experiments, we also observed that irisin promoted capillary-like tube formation of HUVECs (Fig. 3c). These data indicated that irisin improves angiogenesis in heart post-MI.

Irisin stimulated angiogenesis through regulating the ERK signal pathway

ERK signaling plays a critical role in angiogenesis in the post-MI heart, which was also reported to regulate HUVEC proliferation [24]. We investigated whether the ERK signal pathway is involved in irisininduced angiogenesis. In HUVECs, we found that irisin increased the phosphorylation levels of ERK, which was inhibited by U0126 (Fig. 4a). Adult mice were subjected to MI injury and then divided into four groups: MI + PBS, MI + U0126, MI + irisin, and MI + irisin + U0126. Heart tissue was harvested at 7 days post-Ml, and Western blotting analysis was performed. The data showed that irisin treatment increases ERK phosphorylation, which can be blocked by the ERK inhibitor U0126 (Fig. 4b) in accordance with the in vitro results. Endothelial cell migration is essential to angiogenesis [27]. In transwell migration assays, irisin treatment significantly increased HUVEC migration compared with the control, which was repressed 
a1

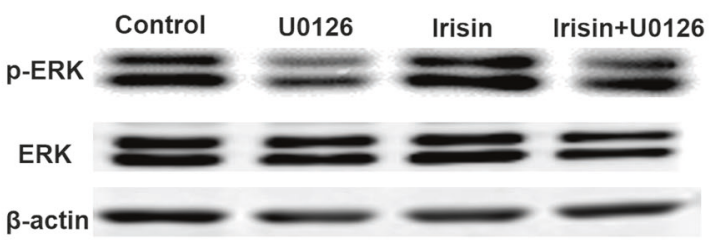

b1

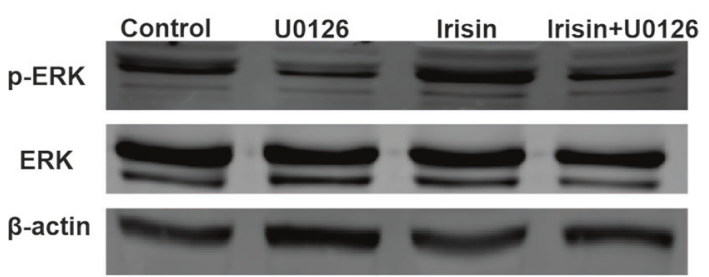

c1
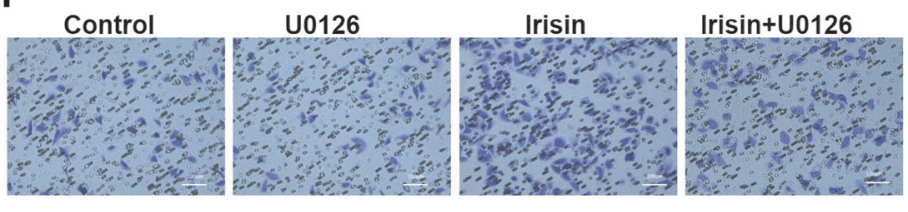

d1
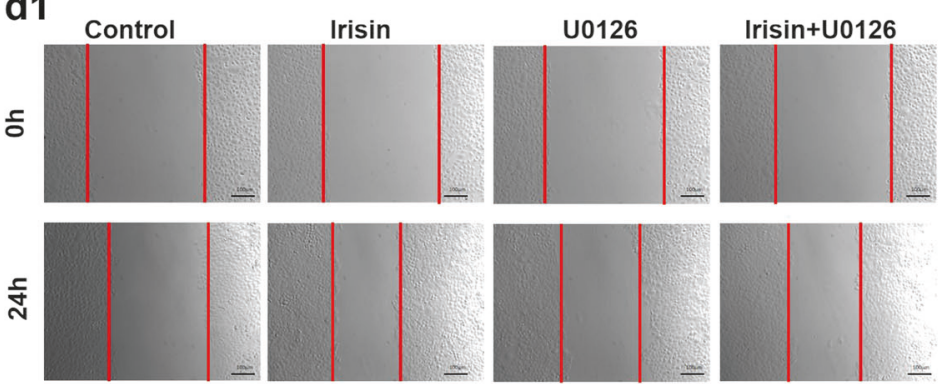

a2
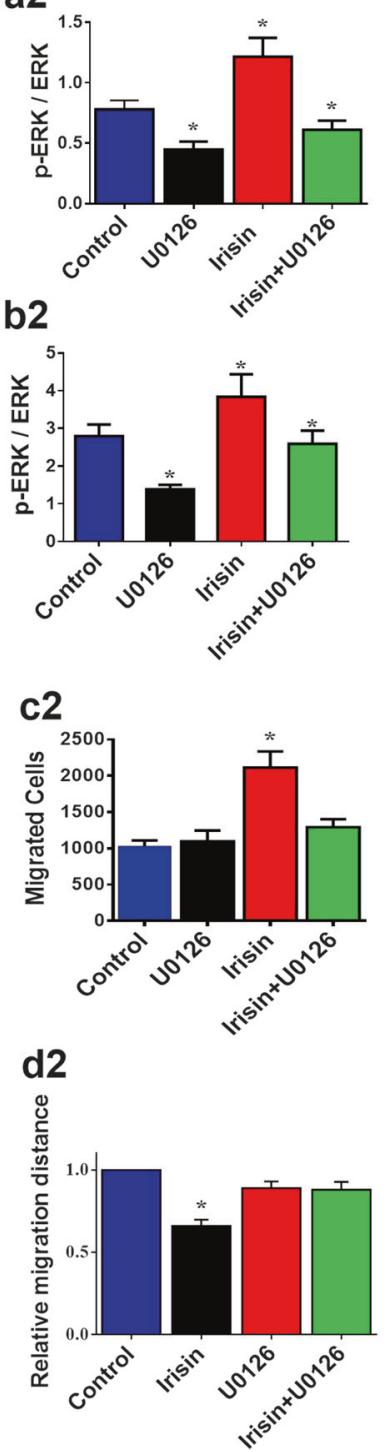

Fig. 4 The pro-angiogenic effect of irisin was associated with the ERK signaling pathway. a Representative images and quantification of phosphorylated and total ERK protein expression in HUVEC analyzed by Western blotting. The ERK inhibitor U0126 was used to block ERK phosphorylation, and $\beta$-actin was used as the internal control. $n=5$. $\mathbf{b}$ Representative images and quantification of phosphorylated and total ERK protein expression in MI mice analyzed by Western blotting. $n=5$. c Representative images and quantification of HUVEC migration detected by transwell migration assay. $n=3$, scale bar $=100 \mu \mathrm{m}$. d Representative images and quantification of HUVEC migration detected by scratch wound-healing assay. $n=3$, scale bar $=100 \mu \mathrm{m} .{ }^{*} P<0.05$ vs. control

by U0126 (Fig. 4c). Meanwhile, the scratch wound healing assay also demonstrated that HUVEC migration was significantly stimulated by irisin, which was partially blocked by U0126 (Fig. 4d). These data indicated that the irisin-induced angiogenesis might be dependent on ERK signal pathway activation.

\section{DISCUSSION}

In the present study, we demonstrated that recombinant irisin exerts a therapeutic effect on $\mathrm{Ml}$ injury in mice through reducing cardiomyocyte apoptosis and fibrosis and promoting angiogenesis in heart. The pro-angiogenic effect of irisin is associated with the ERK signaling pathway.

Irisin is a type of polypeptide that contains 112 amino acids and is mainly secreted by skeletal muscle during exercise [28]. Previous studies have found that irisin plays an important role in metabolism, oxidative stresses and thermogenin expression in different organs $[11,29]$. Irisin drives the change of brown adipose tissue into white adipose tissue; thus, irisin can largely increase energy expenditure [29]. In liver, irisin is associated with hepatic fibrogenesis and extracellular matrix deposition [30]. Our previous study showed that irisin facilitates remote ischemic preconditioning-mediated protective effects against pulmonary I/R injury [12]. In hearts, we [31] and others [7] indicated that irisin reduces myocardial apoptosis and preserves mitochondrial function during I/R injury. However, the therapeutic effect of irisin on $\mathrm{MI}$ has yet to be determined. In the present study, the data confirmed that irisin reduced cardiomyocyte apoptosis post-MI, which is consistent with the findings of a previous study of $\mathrm{I} / \mathrm{R}$ injury [7]. Our previous study showed that irisin protects heart against ischemia-reperfusion injury involving mitochondriadependent apoptosis [31]. Our data also showed that irisin 
reduced noncardiomyocyte apoptosis and significantly promoted angiogenesis and arteriogenesis, which also contributed to the therapeutic effect of irisin against MI injury. Previous studies have reported that irisin treatment decreases endothelial cell apoptosis through the AMPK-PI3K-Akt-eNOS signaling pathway [10].

Angiogenesis plays an important role in saving ischemic cardiomyocytes and promoting cardiac function [32]. However, natural angiogenesis post-MI is insufficient, thus stimulating angiogenesis is an important strategy for MI patients [33]. Several interventions targeting angiogenesis, such as VEGF [34], microRNA-210 [35], glycogen synthase kinase-3 inhibitor [36], apelin [37], and low-intensity pulsed ultrasound [38], rescue cardiac dysfunction post MI. Paracrine factors of mesenchymal stem cells $[39,40]$ also ameliorate post-MI injury partially by promoting angiogenesis. In the present study, we demonstrated that irisin significantly induced angiogenesis, which is consistent with previous studies that suggested that irisin could induce capillary-like tube formation of HUVECs and angiogenesis in zebrafish embryos [41]. However, the underlying mechanism of irisin-induced angiogenesis was unclear. Angiogenesis is based on endothelial cell proliferation and migration to form new vessels through sprouting in the hypoxic or ischemic region [32]. Therefore, endothelial cell proliferation and migration is vital in angiogenesis after MI $[32,39,40]$. The ERK signal pathway plays an important role in endothelial proliferation and migration [42, 43]. ERK-MAPK signaling could promote endothelial cell survival and sprouting during angiogenesis [42]. Interestingly, the ERK signal pathway can also be activated by irisin. In our experiments, we found that irisin induced HUVEC migration, which was dependent on ERK activity. The underlying mechanism of how irisin regulates ERK is currently unknown. Some studies have shown that irisin directly binds to the cell membrane and phosphorylates ERK [28]. A recent study found that irisin bound to the $a V / \beta 5$ integrin receptor on osteocytes, thereby influencing osteocytic osteolysis and bone remodeling [44]. Whether irisin regulates ERK through some receptor and the involved signal transduction requires further study.

In conclusion, we demonstrate that irisin is a myokine that reduces infarct size and improves cardiac function in post-Ml hearts. The therapeutic effect is associated with its pro-angiogenic effect, which likely occurs through an ERK-dependent pathway. Administration of irisin represents potential therapeutic intervention for Ml injury.

\section{ACKNOWLEDGEMENTS}

These studies were supported in part by grants from the National Natural Science Foundation of China $(31730043,31771297)$, National Key R\&D Program of China (2018YFC1312700) Program of Innovative Research Team by National Natural Science Foundation of China (81721001), and Program for Changjiang Scholars and Innovative Research Team in University of Ministry of Education of China (IRT1216).

\section{AUTHOR CONTRIBUTION}

WEW and CYZ designed the research. QL and SQ performed the experiments. LXT, LPL and SQ contributed new reagents or analytic tools. QL and DFH analyzed the data. SQ and QL wrote the draft article. WEW and CYZ revised the article.

\section{ADDITIONAL INFORMATION}

Competing interests: The authors declare no competing interests.

\section{REFERENCES}

1. Vagnozzi RJ, Molkentin JD, Houser SR. New myocyte formation in the adult heart: endogenous sources and therapeutic implications. Circ Res. 2018;123:159-76.

2. Maliken BD, Kanisicak O, Karch J, Khalil H, Fu X, Boyer JG, et al. Gata4-dependent differentiation of $\mathrm{c}^{-\mathrm{Kit}^{+}}{ }^{+}$-derived endothelial cells underlies artefactual cardiomyocyte regeneration in the heart. Circulation. 2018;138:1012-24.
3. Calvert JW, Condit ME, Aragon JP, Nicholson CK, Moody BF, Hood RL, et al. Exercise protects against myocardial ischemia-reperfusion injury via stimulation of beta(3)-adrenergic receptors and increased nitric oxide signaling: role of nitrite and nitrosothiols. Circ Res. 2011;108:1448-58.

4. Li X, Fang W, Hu Y, Wang Y, Li J. Characterization of fibronectin type III domaincontaining protein 5 (FNDC5) gene in chickens: cloning, tissue expression, and regulation of its expression in the muscle by fasting and cold exposure. Gene. 2015;570:221-9.

5. Bashar SM, Samir El-Sherbeiny SM, Boraie MZ. Correlation between the blood level of irisin and the severity of acute myocardial infarction in exercise-trained rats. J Basic Clin Physiol Pharmacol. 2018;30:59-71.

6. Han L, Liu B, Chen X, Chen H, Deng W, Yang C, et al. Activation of Wnt/betacatenin signaling is involved in hair growth-promoting effect of $655-\mathrm{nm}$ red light and LED in in vitro culture model. Lasers Med Sci. 2018;33:637-45.

7. Wang $\mathrm{H}$, Zhao YT, Zhang S, Dubielecka PM, Du J, Yano $\mathrm{N}$, et al. Irisin plays a pivotal role to protect the heart against ischemia and reperfusion injury. J Cell Physiol. 2017;232:3775-85.

8. Zhang K, Li M, Huang H, Li L, Yang J, Feng L, et al. Dishevelled1-3 contribute to multidrug resistance in colorectal cancer via activating Wnt/beta-catenin signaling. Oncotarget. 2017;8:115803-16.

9. Zhao YT, Wang J, Yano N, Zhang LX, Wang $H$, Zhang $S$, et al. Irisin promotes cardiac progenitor cell-induced myocardial repair and functional improvement in infarcted heart. J Cell Physiol. 2019;234:1671-81.

10. Lu J, Xiang G, Liu M, Mei W, Xiang L, Dong J. Irisin protects against endothelial injury and ameliorates atherosclerosis in apolipoprotein E-Null diabetic mice. Atherosclerosis. 2015;243:438-48.

11. Zhu D, Wang H, Zhang J, Zhang X, Xin C, Zhang F, et al. Irisin improves endothelial function in type 2 diabetes through reducing oxidative/nitrative stresses. J Mol Cell Cardiol. 2015;87:138-47.

12. Chen $\mathrm{K}, \mathrm{Xu} Z$, Liu Y, Wang Z, Li Y, Xu X, et al. Irisin protects mitochondria function during pulmonary ischemia/reperfusion injury. Sci Transl Med. 2017;9 pii: eaao6298.

13. Nakada $Y$, Canseco D, Thet $S$, Abdisalaam S, Asaithamby A, Santos $C$, et al. Hypoxia induces heart regeneration in adult mice. Nature. 2017;541:222-7.

14. Reza MM, Sim CM, Subramaniyam N, Ge X, Sharma M, Kambadur R, et al. Irisin treatment improves healing of dystrophic skeletal muscle. Oncotarget. 2017;8:98553-66.

15. Wang A, Zhang H, Liang Z, Xu K, Qiu W, Tian Y, et al. U0126 attenuates ischemia/ reperfusion-induced apoptosis and autophagy in myocardium through MEK/ERK/ EGR-1 pathway. Eur J Pharmacol. 2016;788:280-5.

16. Wang W, Li L, Xia X, Fu W, Liao Q, Lan C, et al. Dedifferentiation, proliferation, and redifferentiation of adult mammalian cardiomyocytes after ischemic injury. Circulation. 2017;136:834-48.

17. Yang D, Fu W, Li L, Xia X, Liao Q, Yue R, et al. Therapeutic effect of a novel Wnt pathway inhibitor on cardiac regeneration after myocardial infarction. Clin Sci. 2017;131:2919-32.

18. Wang WE, Yang D, Li L, Wang W, Peng Y, Chen C, et al. Prolyl hydroxylase domain protein 2 silencing enhances the survival and paracrine function of transplanted adipose-derived stem cells in infarcted myocardium. Circ Res. 2013;113:288-300.

19. Wang N, Chen C, Yang D, Liao Q, Luo H, Wang X, et al. Mesenchymal stem cellsderived extracellular vesicles, via miR-210, improve infarcted cardiac function by promotion of angiogenesis. Biochim Biophys Acta Mol Basis Dis. 2017;1863:2085-92.

20. Mohamed T, Ang Y, Radzinsky E, Zhou P, Huang $Y$, Elfenbein A, et al. Regulation of cell cycle to stimulate adult cardiomyocyte proliferation and cardiac regeneration. Cell. 2018;173:104-16. e12.

21. Zheng G, Li H, Zhang T, Yang L, Yao S, Chen S, et al. Irisin protects macrophages from oxidized low density lipoprotein-induced apoptosis by inhibiting the endoplasmic reticulum stress pathway. Saudi J Biol Sci. 2018;25:849-57.

22. Zhang $Y$, Song $H$, Wu F, Mu Q, Jiang $M$, Wang F, et al. Irisin inhibits atherosclerosis by promoting endothelial proliferation through microRNA126-5p. J Am Heart Assoc. 2016;5 pii: e004031.

23. Qiao X, Nie Y, Ma Y, Chen Y, Cheng R, Yin W, et al. Irisin promotes osteoblast proliferation and differentiation via activating the MAP kinase signaling pathways. Sci Rep. 2016;6:18732.

24. Song $H$, Wu $F$, Zhang $Y$, Wang $F$, Jiang $M$, Wang $Z$, et al. Irisin promotes human umbilical vein endothelial cell proliferation through the ERK signaling pathway and partly suppresses high glucose-induced apoptosis. PLoS ONE. 2014;9: e110273.

25. Wu F, Song $H$, Zhang $Y, M u ~ Q$, Jiang $M$, Wang $F$, et al. Irisin induces angiogenesis in human umbilical vein endothelial cells in vitro and in zebrafish embryos in vivo via activation of the ERK signaling pathway. PLoS ONE. 2015;10:e0134662.

26. Wang M, Chen DQ, Chen L, Liu D, Zhao H, Zhang ZH, et al. Novel RAS inhibitors poricoic acid ZG and poricoic acid ZH attenuate renal fibrosis via a Wnt/betacatenin pathway and targeted phosphorylation of smad3 signaling. J Agric Food Chem. 2018:66:1828-42. 
27. Lamalice L, Le Boeuf F, Huot J. Endothelial cell migration during angiogenesis. Circ Res. 2007;100:782-94.

28. Zhang Y, Li R, Meng Y, Li S, Donelan W, Zhao Y, et al. Irisin stimulates browning of white adipocytes through mitogen-activated protein kinase p38 MAP kinase and ERK MAP kinase signaling. Diabetes. 2014;63:514-25.

29. Bostrom P, Wu J, Jedrychowski MP, Korde A, Ye L, Lo JC, et al. A PGC1-alphadependent myokine that drives brown-fat-like development of white fat and thermogenesis. Nature. 2012;481:463-8.

30. Petta S, Valenti L, Svegliati-Baroni G, Ruscica M, Pipitone RM, Dongiovanni P, et al. Fibronectin type III domain-containing protein 5 rs3480 A>G polymorphism, irisin, and liver fibrosis in patients with nonalcoholic fatty liver disease. J Clin Endocrinol Metab. 2017;102:2660-9.

31. Wang Z, Chen K, Han Y, Zhu H, Zhou X, Tan T, et al. Irisin protects heart against ischemia-reperfusion injury through a SOD2-dependent mitochondria mechanism. J Cardiovasc Pharmacol. 2018;72:259-69.

32. Albrecht-Schgoer K, Schgoer W, Holfeld J, Theurl M, Wiedemann D, Steger C, et al. The angiogenic factor secretoneurin induces coronary angiogenesis in a model of myocardial infarction by stimulation of vascular endothelial growth factor signaling in endothelial cells. Circulation. 2012;126:2491-501.

33. Oostendorp M, Douma K, Wagenaar A, Slenter JM, Hackeng TM, van Zandvoort $M A$, et al. Molecular magnetic resonance imaging of myocardial angiogenesis after acute myocardial infarction. Circulation. 2010;121:775-83.

34. Zhu $H$, Jiang $X$, Li X, Hu M, Wan W, Wen $Y$, et al. Intramyocardial delivery of VEGF165 via a novel biodegradable hydrogel induces angiogenesis and improves cardiac function after rat myocardial infarction. Heart Vessels. 2016:31:963-75.

35. Arif M, Pandey R, Alam P, Jiang S, Sadayappan S, Paul A, et al. MicroRNA-210mediated proliferation, survival, and angiogenesis promote cardiac repair post myocardial infarction in rodents. J Mol Med. 2017;95:1369-85.
36. Baruah J, Hitzman R, Zhang J, Chaudhuri S, Mastej V, Wary KK. The allosteric glycogen synthase kinase-3 inhibitor NP12 limits myocardial remodeling and promotes angiogenesis in an acute myocardial infarction model. J Biol Chem. 2017;292:20785-98.

37. Hou X, Zeng H, He X, Chen JX. Sirt3 is essential for apelin-induced angiogenesis in post-myocardial infarction of diabetes. J Cell Mol Med. 2015;19:53-61.

38. Shindo T, Ito K, Ogata T, Hatanaka K, Kurosawa R, Eguchi K, et al. Low-intensity pulsed ultrasound enhances angiogenesis and ameliorates left ventricular dysfunction in a mouse model of acute myocardial infarction. Arterioscler Thromb Vasc Biol. 2016;36:1220-9.

39. Luo L, Tang J, Nishi K, Yan C, Dinh P, Cores J, et al. Fabrication of synthetic mesenchymal stem cells for the treatment of acute myocardial infarction in mice. Circ Res. 2017;120:1768-75.

40. Wang N, Chen C, Yang D, Liao Q, Luo H, Wang X, et al. Mesenchymal stem cellsderived extracellular vesicles, via miR-210, improve infarcted cardiac function by promotion of angiogenesis. Biochim Biophys Acta. 2017;1863:2085-92.

41. Wu $F$, Song $H$, Zhang $Y$, Zhang $Y, M u ~ Q$, Jiang $M$, et al. Irisin induces angiogenesis in human umbilical vein endothelial cells in vitro and in zebrafish embryos in vivo via activation of the ERK signaling pathway. PLoS ONE. 2015;10:e0134662.

42. Mavria G, Vercoulen $Y$, Yeo $M$, Paterson $H$, Karasarides $M$, Marais $R$, et al. ERKMAPK signaling opposes Rho-kinase to promote endothelial cell survival and sprouting during angiogenesis. Cancer Cell. 2006;9:33-44.

43. Chamorro-Jorganes $A$, Lee $M$, Araldi E, Landskroner-Eiger $S$, Fernández-Fuertes $M$, Sahraei M, et al. VEGF-induced expression of miR-17-92 cluster in endothelial cells is mediated by ERK/ELK1 activation and regulates angiogenesis. Circ Res. 2016;118:38-47.

44. Kim H, Wrann CD, Jedrychowski M, Vidoni S, Kitase $Y$, Nagano K, et al. Irisin mediates effects on bone and fat via alphaV Integrin receptors. Cell. 2018;175: 1756-68. e17 